\title{
Enhancing Business Process Modeling with Context and Ontology
}

\author{
Jamal EL BOUROUMI, Hatim GUERMAH, Mahmoud NASSAR \\ IMS Team, ADMIR Laboratory \\ Rabat IT Center, ENSIAS, Mohammed V University in Rabat, Morocco
}

\begin{abstract}
Business process is a sequence of events and tasks that encompass actions and people. Therefore, a company that pays much attention to its business processes has to clearly identify and define the procedures of their relations. However, with the exponential evolution in ubiquitous computing, the exploitation of information spread all over different devices has become essential to further improve business processes. Hence, in this paper we present a new approach for business process modeling that is based on context-awareness and ontology. We propose a set of meta-model for the elements that we find very important, taking into account the context and, in modeling, the business process. To validate our approach, we propose a concrete case study about transport system to provide a proof of the applicability as well as the utility of the model.
\end{abstract}

Keywords-Business process; business process modeling; context; context-awareness; ontology

\section{INTRODUCTION}

Companies are known by their constant search for competitive advantages in order to create new business opportunities and gain new areas of markets. Thus, they are permanently in need to perform digital transitions and continuous improvements which can only be achieved through implementing technology. One of the most known transformation is Business Processes that were introduced to help companies to regain their lost positions and powers. The use of Business Processes has a huge impact because it brings into existence new kinds of process-oriented organizations in which the structural hierarchy degraded. Under processoriented organizations the contributors are assigned tasks and accomplishments which are sorted accurately to avoid any unexpected problems. Business Processes has made significant results in increasing the performance indicators (KPI, related to time, cost and quality of the organizations). So, the modeling of business processes is at the centre of an organization's analysis process. Whether as part of a global reorganization or a targeted improvement approach. Process modeling formalizes the function of an organization. It involves structuring and representing the activities of an organization, by using a graphical notation to visually represent the flow of activities. Modeling is based on specialized methods and tools as well as implements process reference frameworks.

In recent years, several researchers have proposed approaches for business process modelling.

It also displays new trends in ICT and the ubiquitous computing. The development of context-awareness service is a challenge in current research projects. In fact, since its appearance in the 1990s, several researchers have proposed many approaches to meet the challenge of context awareness, especially with the birth of service-oriented architectures.

However, several research questions remain to be addressed. This is due to a series of reasons, in particular the modeling of contextual information and the consideration of context in the business processes modelling.

However, our work allows to model contextual information, integrating this model in the modeling of business processes in order to obtain a contextual business process. With the importance and value that the semantic aspect gives to the model, we will present a semantic model of the contextual business process model based on ontology.

In this article, we integrate the context into the BP as follows:

- We define the context by the proposal of a context and sensor metamodel.

- We define a business process model that takes into account the context.

- We enrich this model by adding a semantic aspect.

- We apply our approach to a case study.

The rest of this paper is organized as follows: We start with a background and related works where we present some definitions and the state of the art on the BP modeling and the context. Section three presents the different models of our approach as well as the case study of transport. In section four we give a simple conclusion and a vision of our future work.

\section{BACKGROUND}

\section{A. Context}

The notion of context has been a research subject for years, and until now, there is no general and standard definition. Apparently, several researchers have tried to give a definition, we are citing the most widely used definitions in the scientific community.

Let's start with the definition of [30] which categorized the context into three types, location, the identity of the person and the object in question. [31] defines the context as having three basic elements, the time, the date and the changes in that period.

But the most used definition, on which we rely is the definition of Dey et $\mathrm{Al}$ [32], it defines the context as follows: 
"Context is any information used to characterize the situation of an entity. An entity may be a person, place or object considered relevant for the interaction between a user and an application, including the user and the application itself."

\section{B. Context-Awareness}

Regarding context Awareness, several visions are proposed to define a context-aware system. [1] Considers contextaware applications as "applications whose behaviour may vary depending on the context". Its applications must automatically extract information and perform actions based on the context of the user detected by the sensors. Similarly, [2] perceives context Awareness as the ability to adapt the functioning of a system in order to increase its usability and efficiency by taking into account the context of the user. Another interesting vision is provided by [3] which makes it possible to link the sensitivity and the context to the service offered by the application or the system. In fact, a system is said to be context-aware if it can automatically change these forms of services or if it is able to trigger a service as a response to changing the value of an information or a set of information which characterize the service.

These definitions may be considered limited, according to [4], since they exclude applications that use only the context and that do not make any adaptation of context-aware systems. To generalize these definitions, [4] considers that a system is context-aware if it uses context information to make information or / and services useful to the user available and considers that this utility is dependent of the user's task. This definition is also limited since a context-aware system deals with other aspects other than the context acquisition component, such as the interpretation of the context and the necessary adaptations. To overcome these shortcomings, [5] perceives that a system's sensitivity to context "is its ability to acquire, manage, interpret, and respond to changes in the context in order to provide the appropriate service". Thus we can consider that the two definitions of [4] and [5] complement one another and can be considered as reference to define a context-aware system.

\section{Context-Awareness}

For [6] ontology is the assumptions we make about the kind and nature of reality and what exists. Ontology is also defined as the nature of the world and what we can know about it.

In the field of computer science, ontology for [7] refers to "an explicit specification of a conceptualization" or a more refined way while [8] sees it as a "partial and formal specification of a shared conceptualization". Ontology is among the most used tools of the Semantic Web and Artificial Intelligence. It allows to model resources based on conceptual representations of the domain under study and allows systems and applications to draw inferences from them.

\section{Business Process}

A business process is a sequence of events that encompasses actions, people and the sequence of work. This concept comes from production lines but applies also to commercial transactions. Whatever the structure of a manufacturing, service or sales organization, it is underpinned by its business processes.
In the literature, several definitions are available for the Business Process. [9] Defines the business process as "a structured, measured set of activities and flows that use necessary resources of the organization to provide specified output for a particular customer". [10] Defines it as follows "the combination of a set of activities within an enterprise with a structure describing their logical order and dependence whose objective is to produce a desired result". [11] Defines a process as a productive activity which includes working for something, moving of people, materials, information and interaction.

\section{RELATED WORK}

Business process modeling is a major challenge for all companies that aim to understand and improve their business. With the emergence of ubiquitous computing, consideration of the context has become paramount in the BP. So, in this section, we will present some approaches that are related to our work.

Modeling contextual information consists of representing the context in a well-defined format that is easily shared and used by the various stakeholders of the application. However, several approaches have been proposed to standardize the Context Modeling: [12][13] model the context as keys/value or each key defines the contextual information so the value presents its value, [14][15][16]are based on mark-up language, it is a more structured approach that presents information in a hierarchical structure, [17]presents the context in an objectoriented way, so the contextual information is encapsulated in an object in order to model it in a ubiquitous computing,[18] model is based on MDA, it allows for a model that is based on MOF (Meta-model facility) and[19][20] use ontology to model contextual information in a declaration form, so it allows for a semantic reasoning in these information.

Also for business process modeling, several methods and languages are proposed such as UML which allows for a modeling of any system in an object-oriented way, EPC (Event driven chains), IDEF0 that allows to model an organization's actions, Petri Net which is a mathematical graphical method to present systems, PSL (Process Specification Language) [21] that allows to represent model business process based on an ontology, etc. Thus, BPMN which is the standard OMG for business process modeling simplifies the understanding as well as manipulates and improves the quality of a business process.

In order to integrate the context in business process modeling several approaches have been proposed, including those based on the methods mentioned above, to model the context, the BP and other approaches that invent new methods. [22] extends BPMN meta-model in order to add elements (task, collector) to represent contextual elements. [23] has proposed business process and context meta-models, [24] is a layered architecture which is proposed to represent the domain, the context and the BP in order to produce a Contextual business process. [25] has proposed an approach to model the contextual business process based on BPMN. [26] proposes a new context model that takes into account the business process situations, Author in [33] has proposed a conceptual method of depicting the context of a business process, in [34] authors has proposed a method based on DMN for modelling business process . 
Other methods have added semantic aspects to business processes: [27] have proposed an ontology of BPMN language that called BPMN Ontology, it allows to represent BPMN in the form of OWL-DL. [28] has proposed a framework that are called BPAL to manage ontologies based on BP modeling concepts (processes, atoms etc.).

\section{CAse Study: Transport System}

The system we have chosen as a case study is an example of an informational system made by a transport company that suggests the best routes in Rabat city (MyCity App). Indeed, MyCity is an application of transport services meant for residents and passengers of the city of rabat. A user of this application is anyone who wishes to have a vision on a journey inside the city of Rabat.

On the other hand, the current applications don't provide information about transports timetables and users' information (preferences, history, etc.) in order to make the service appropriate to the user. As a result, the services provided by these applications and the routes offered are not optimized for the users in question.

Indeed, a typical transport application includes, but is not limited to, the following services:

- Search Trip: the user enters his destination and,( if he wants, his location) otherwise, his location will be automatically collected by the sensory system.

- Display results: The system displays the different possible routes and orders them according to the history (preferred means of transport/departure time, etc.) and the user preferences (user context).

- Choose route: the user must choose the preferred route.

- Display route: the system must display the path, it must take into account the configuration of the user's device and it must follow the path with him in real time.

Depending on the scenario, several challenges arise:

- Context capture from various sources (user context, device, etc.).

- Considers the user's context and the context of transport in the various services (journey search, journey display, etc.).

Indeed, all these services and challenges are implemented in our system.

\section{The Proposed ApProach}

Computers, smartphones, smart sensors are occupying more and more space in our life. However, the amount of information that circulates on these devices can help us in the enrichment of several systems that we will need daily, such as transport, health, telecommunication, sport and any system or service. Therefore, our idea is to take advantage of all this data (which is voluminous and rich) to improve the quality of services for users. As Mark Weiser says: "There is more information available at our fingertips during a walk in the woods than in any computer system, yet people find a walk among trees relaxing and computers frustrating. Machines that fit the human environment, instead of forcing humans to enter theirs, will make using a computer as refreshing as taking a walk in the woods".

However, with the place that Business Processes takes in the strategy of companies, and with this area of ubiquitous computing, the integration of contextual information into BP will increasingly improve the quality of service for end-users and provide a clearer vision for management to make the right decisions.

Our approach as in Fig. 1 is based on several components/models:

- Sensors: it is the model which is responsible for the representation of the sensors.

- Context: allows to model contextual information.

- CBPM: Contextual Business Process Model, it is the model that presents business process.

- CBPM Ontology: Contextual Business Process Model Ontology, it is an ontology that corresponds to the CBPM model.

Therefore, the ultimate objective of the proposed approach is to provide a general framework for modeling the context and to integrate it into the modeling of business processes, then add a semantic aspect to this model by proposing an ontology that corresponds to the proposed model.

Indeed, by the proposal of these models we were able to model the most important elements of the context and the BP, and to also bring them together in a single model that is CBPM which simplified the use of context in the execution of the BP model. This modeling approach facilitates the integration and the re-use of one or more models of our approach by other related systems / applications.

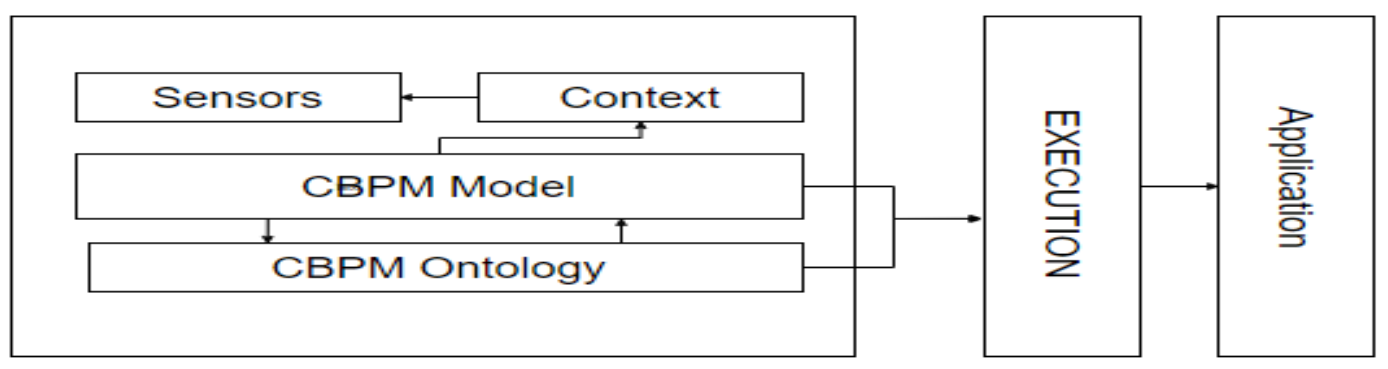

Fig. 1. The Proposed Approach. 


\section{A. Sensors}

Sensors are components that are responsible for extracting contextual information. A sensor is any element capable of returning information about the user and the environment.

In general, there are two types of sensors: logical and physical:

- Physical sensors: they are the materials that can extract information related to the context of the organization, the user or the business process itself. Like GPS, localization, the state of the execution of the business process etc.

- Logical Sensors: they are intelligent programs that allow to retrieve information from the different possible sources: web services, user calendar, reasoning about user preferences.

In order to properly represent the different Sensors, we present in Fig. 2 a simplified meta-model of sensor. Fig. 3 presents the application of this meta-model to our scenario of transport system presented above. Among the different sensors related to this field, we have chosen the following sensors:

- Location Sensor: it is a physical sensor that captures the location of the user.

- Preference Sensor: it is a logical sensor that allows us to reason about the user's history in order to obtain his preferences.

\section{B. Context}

This model makes it possible to represent the context in a well-structured format in order to use it by other components. For the context in the Business process, it is any information that can give an added value to the modeling, execution or improvement of the BP.
So, and in order to give a generic formalization to the context that will be usable in any application, we propose in Fig. 4 a context metamodel based on the work [29].

Our meta-model is composed of the following elements:

- Context: represents the context, it is composed of sub Contexts.

- Sub Context: represents a sub-context, it is composed of Property context.

- Context Property: it is the contextual element; it can be attribute or Context Relationship.

- Sensor: represents the context provider.

- Context Relationship: represents the relationships between the different elements of the context.

- Attribute: is an atomic value that represents contextual information.

- Validity: represents the period of validity of a contextual information.

According to our scenario, we present in the Fig. 5 the context of our transport system, it is divided into the following sub Context:

- Use Context: Contains the attributes that describe the user's context (location, Preference).

- Environment Context: Contains the attributes of the environment.

Device Context: Contains the attributes which describe the device information (screen size, battery, ram, ...).

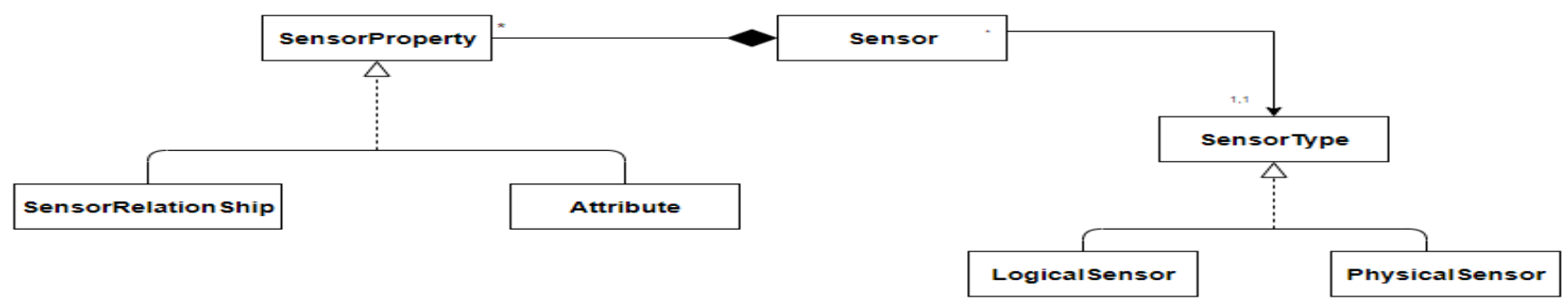

Fig. 2. Sensors Meta-Model.
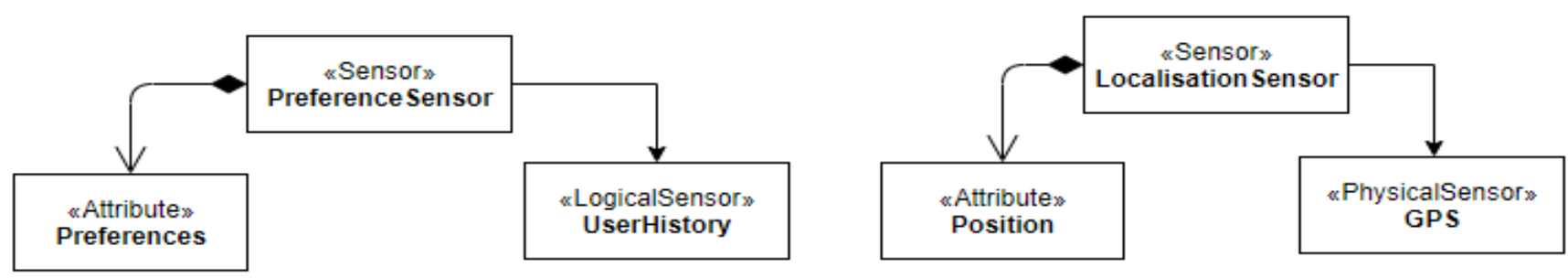

Fig. 3. Transport Sensors. 


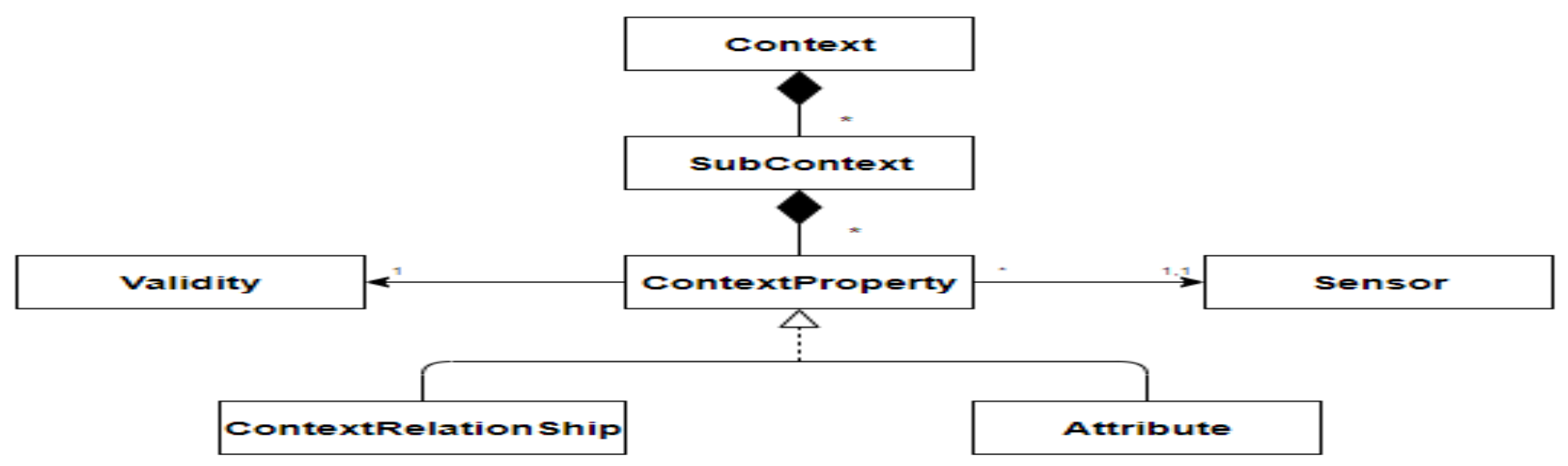

Fig. 4. Context Meta-Model.

\section{CBPM Model}

Taking the context into account in the business process significantly increases its representation and the productivity of the organization. And to respond to the different challenges of the BP, we have proposed the meta-model presented in Fig. 6 which represents the most important aspects of a business process, and it models the integration of context in this BP.

Our business process meta-model is composed of the following elements:

- Process: represent the business process.

- Sub Process: represents a subset of processes, it is composed of Process Element.

- Process Element: represents the main elements of a business process, it can be Gateway, Event or Service.

- Service: represents the element of Process which are services, in general these are the services offered by the organization.

- Event: represents the elements that can be triggered before, during or after the execution of the business Process.

- Gateway: it is a connection between the different services and events.

- Data: represents the data used in the business process, they can be Process Data or Context Data.

- Process Data: represents the process data.
- Context Data: represents contextual information.

Let's apply this meta-model to our case study, according to the scenario our application offers several services such as the search for a route, display of a route... the Fig. 7 presents the Contextual Business Process Model of the transport system.

\section{CBPM Ontology}

In order to enrich the different elements of the CBPM model with a semantic aspect, we have proposed the CBPM Ontology in Fig. 8 which is the result of a transformation of the CBPM model.

CBPM Ontology is made up of three main ontologies:

- Process Ontology: this is the ontology that describes the different elements of BP.

- Context Ontology: this is the ontology that describes the user's context, device and environment.

- Domain Ontology: This is the ontology of the studied domain, like Transport Ontology for the transport domain.

The mapping between the elements of CBPM model and CBPM Ontology is based on the classes and instances of the ontology and the classes and properties of the model CBPM. This mapping allows to give a semantic formalization to our CBPM model.

Fig. 8 presents also the mapping based on the case study described above.

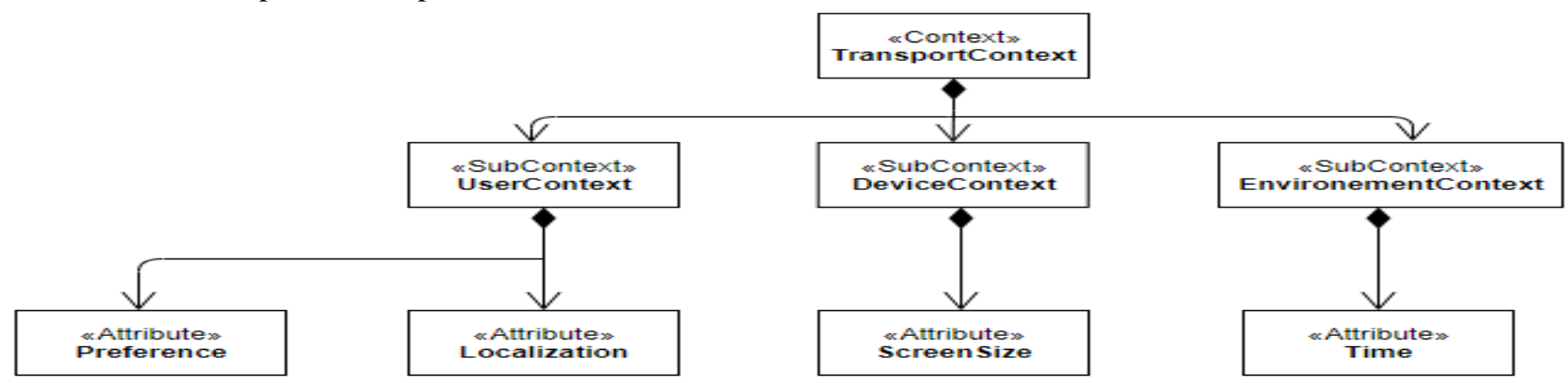

Fig. 5. Transport Context. 


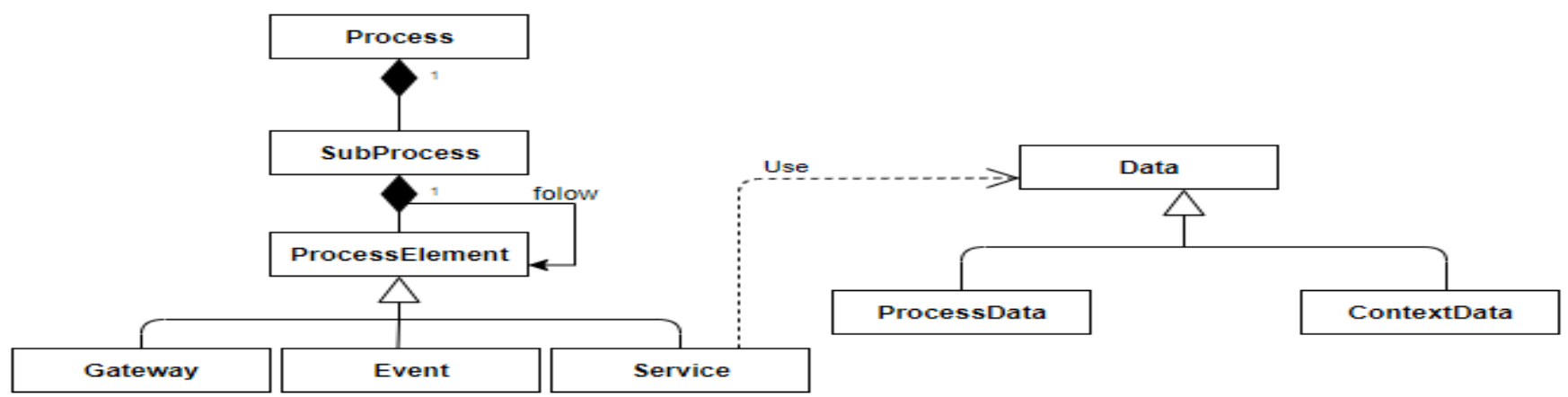

Fig. 6. CBPM Meta-Model.

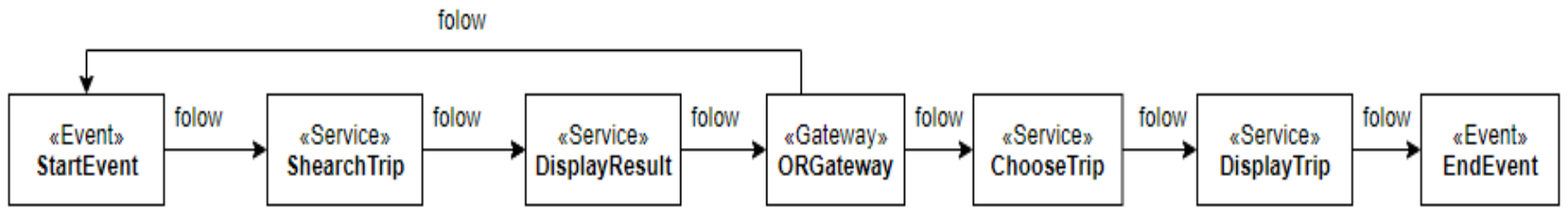

Fig. 7. Transport CBPM.

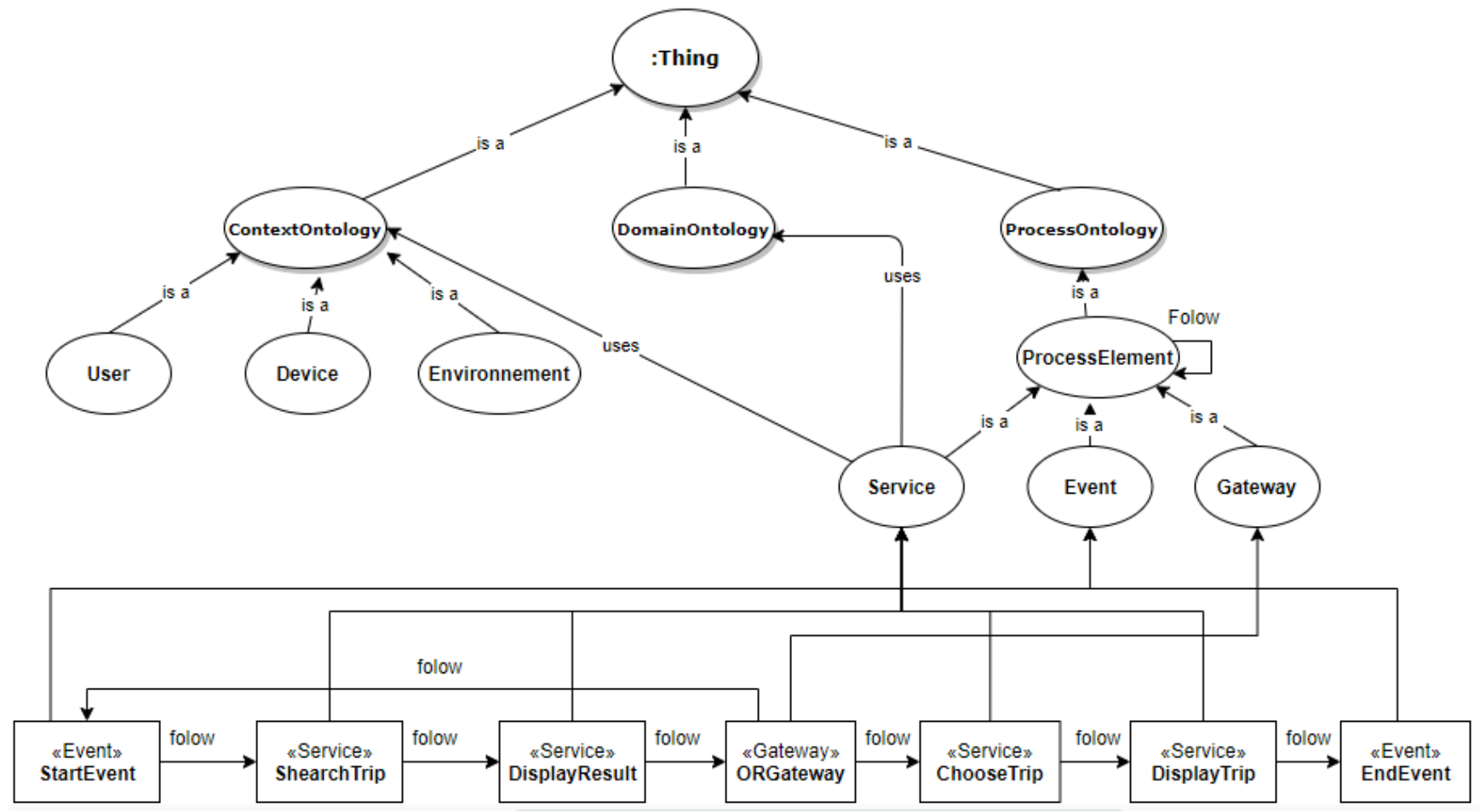

Fig. 8. CBPM Ontology.

\section{RESULT}

Based on the different meta-models presented above, we have created a general framework to model not only the business processes, but also the contextual information, the sensors that allow us to extract this information. CBPM is a model that allows to model the business processes in a generic way independent of the field studied, and also to integrate the notion of context.
As mentioned above, our approach has been designed in such a way that it is easily integrated and reusable globally or partially with other systems. For example, for applications that rely only on the context without BP can use our sensor design model and context.

In fact, in order to validate our approach, we applied it to the transport study case which presents a challenge for the use of contextual information in the implementation of business processes. 
Indeed, from the CBPM Model, we were able to extract the various services and events of our business process as well as the gateways. This has facilitated its execution and the adaptation of these services to the context of the user.

Indeed, for the search Trip service presented in Fig. 9, it allows to launch a search of the best routes between the departure and the destination of the user. This service is based on the geographical position, for the display Result service presented in Fig. 10, it is based on the preferences and the history of the user in order to propose routes.

In fact, all of these services are based on the hardware characteristics of the user's device (size, PC/Mobile).

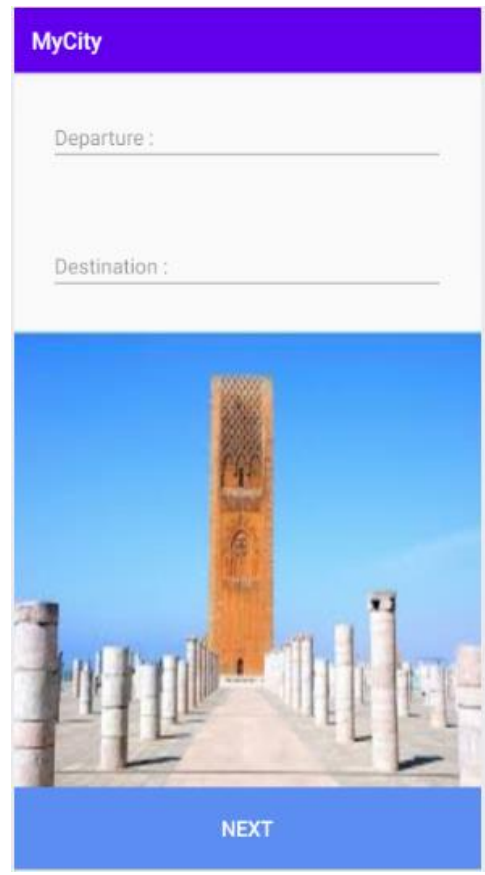

Fig. 9. SearchTrip.

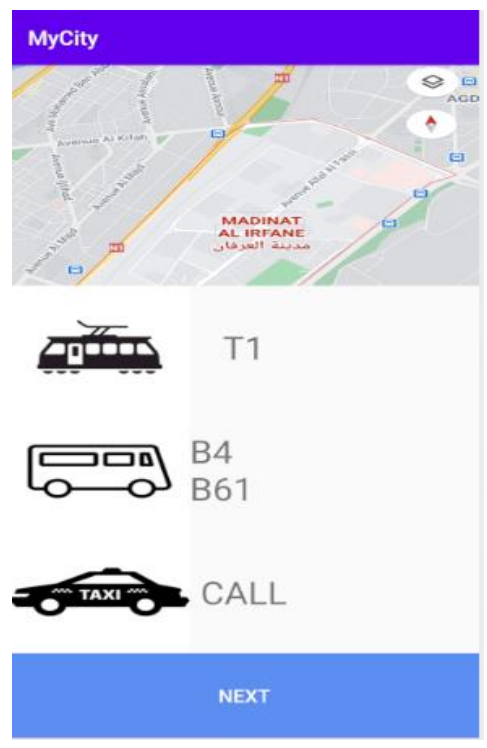

Fig. 10. Display Result.

\section{CONCLUSION}

In this article we have presented a new approach to model the context and the business process. We proposed an architecture with several components, CBPM:

- Sensors: to model sensors according to their different types (logic, physics).

- Context metamodel: a context metamodel to represent contextual information.

- CBPM metamodel: the business process model that takes into account contextual information.

- CBPM Ontology: an ontology which is the result of a transformation of the CBPM model based on the rules for converting meta-models into ontology, which gives the latter a semantic aspect.

In addition, we presented the classical methods and language for business process modelling. We also stated approaches that have responded to the problem of taking context into account in the business process and others that have enriched business models with semantic aspects through approaches like ontology.

In our future work, we will use this model to propose an approach for business process improvement taking into account the different service events gateway presented in the CBPM Model.

\section{REFERENCES}

[1] P. Brown, J. Bovey and X. Chen, Context-aware Applications: from the Laboratory to the Marketplace, IEEE Personal Communications, Vol 4, no 5, pp. 58-64, 1997.

[2] M. Baldauf, S. Dustdar, and F. Rosenberg, A survey on context aware systems, International Journal of Ad Hoc and Ubiquitous Computing, vol. 2, no. 4, pp. 263-277, 2007.

[3] M. Miraoui, and C. Tadj, A service oriented definition of context for pervasive computing, In The 16th International Conference on Computing, IEEE Computer Society, 2007.

[4] A. K. Dey, Supporting the construction of context-aware applications, In Dagstuhl Seminar on Ubiquitous Computing, Dagsthul, Germany, 2001.

[5] W. Xiaohang, The context gateway: a pervasive computing infrastructure for context aware services, Technical report, National university of Singapore, 2003.

[6] RICHARDS, Keith. Qualitative inquiry in TESOL. Springer, 2003.

[7] T.R. Gruber, A translation approach to portable ontology specifications, Knowledge Acquisition, Vol. 5, no. 2, pp. 199-220, 1993.

[8] N. Guarino, Formal ontology, conceptual analysis and knowledge representation, International Journal of Human-Computer Studies, Vol. 43, no. 5/6, pp. 625-640,1995.

[9] Laakso, T. Process Assessment Method - an approach for business process development. In: Plonka F., Olling G. (eds) Computer Applications in Production and Engineering. IFIP - The International Federation for Information Processing. Boston, MA: Springer. 1997.

[10] Aguilar-Saven R.S.. Business process modelling: Review and framework, International Journal of Production Economics. 90: 129149. 2004.

[11] Anttila, J.; Jussila, K. An advanced insight into managing business processes in practice, Total Quality Management 24(8): 918-932. . 2013.

[12] Bettini., 2010: A survey of context modeling and reasoning techniques. Pervasive and Mobile Computing, vol. 6, no 2, pp. 161-180, 2010. 
[13] Strang, A context modeling survey. In Ubicomp, First International Workshop on Advanced Context Modeling, Reasoning and Management, 2004.

[14] Venezia, Pervasive ICT Social Aware Services enablers. 14th International Conference on Intelligence in Next Generation Networks (ICIN). Berlin, Germany, 2010.

[15] Knappmeyer:ContextML: A light-weight context representation and context management schema. 5th IEEE International Symposium on Wireless Pervasive Computing (ISWPC), Modena, Italy, 2010.

[16] Klyne: G. Klyne, F. Reynolds, C. Woodrow, H. Ohto, J. Hjelm, M.H. Butler and L. Tran, Composite Capability/Preference Profiles (CC/PP): Structure and Vocabularies 1.0. W3C Recommendation, 2004.

[17] Cheverest: The role of adaptive hypermedia in a contextaware tourist guide, Communication of ACM, vol. 45 no. 5, pp. 47-51, 2002.

[18] Chihani: Enhancing Existing Communication Services with Context Awareness, Journal of Computer Networks and Communications, Volume 2012.

[19] Ferry: Adaptation Dynamique d'Applications au Contexte en Informatique Ambiante, Research Report I3S , number I3S/RR-200820-FR 2008.

[20] Chen: An ontology for context-aware pervasive computing environments. The Knowledge Engineering Review, vol. 18 no. 3, pp 197-207, 2004.

[21] Gruninger Michael, Schlenoff Craig, "Process Specification Language (PSL):results of the first pilot implementation", Proceedings of IMECE: International Mechanical Engineering Congress and Exposition, pp 1$10,1999$.

[22] Alaaeddine Yousfi, Christine Bauer, Rajaa Saidi, Anind K. Dey, uBPMN: A BPMN extension for modeling ubiquitous business processes, Information and Software Technology, Volume 74,Pages 5568, 2016.

[23] Saidani, Oumaima \& Rolland, Colette \& Nurcan, Selmin. (2015). Towards a Generic Context Model for BPM. 2015. 10.1109/HICSS.2015.494.
[24] Santoro, Flávia \& Baião, Fernanda \& Revoredo, Kate \& Nunes, Vanessa. (2017). Modeling and Using Context in Business Process Management: A Research Agenda. Modélisation et utilisation du contexte. 17. 10.21494/ISTE.OP.2017.0130.

[25] SANTRA, Debarpita et CHOUDHURY, Sankhayan. C-BPMN: A Context Aware BPMN for Modeling Complex Business Process. arXiv preprint arXiv:1806.01333, 2018.

[26] DA CUNHA MATTOS, Talita, SANTORO, Flávia Maria, REVOREDO, Kate, et al. A formal representation for context-aware business processes. Computers in Industry, vol. 65, no 8, p. 1193-1214, 2014.

[27] Marco Rospocher and Chiara Ghidini and Luciano Serafini: An ontology for the Business Process Modelling Notation, FOIS,2014.

[28] De Nicola, Antonio \& Lezoche, Mario \& Missikoff, Michele. (2007). An Ontological Approach to Business Process Modeling. 1794-1813.

[29] Guermah: Context modeling and reasoning for building context aware services. In ACS International Conference on Computer Systems and Applications (AICCSA), IEEE, pp. 1-7, May, 2013.

[30] Bill $N$ Schilit, Marvin $M$ Theimer: Disseminating active map information to mobile hosts. IEEE network, vol. 8, no. 5, pages 22-32, 1994.

[31] Guanling Chen, David Kotzet al: A survey of context-aware mobile computing research. Rapport technique, Technical Report TR2000-381, Dept. of Computer Science, Dartmouth College, 2000.

[32] Anind Dey, Gregory Abowd, Peter Brown, Nigel Davies, Mark Smith \& Pete Steggles. Towards a better understanding of context and contextawareness. In Handheld and ubiquitous computing, pages 304307. Springer, 1999.

[33] Zhao, Xiaohui, et al. "Enabling intelligent business processes with context awareness." 2018 IEEE International Conference on Services Computing (SCC). IEEE, 2018.

[34] Song, Rongjia, et al. "A DMN-based method for context-aware business process modeling towards process variability." International Conference on Business Information Systems. Springer, Cham, 2019. 